\title{
Dynamic patterns of compaction in brittle porous media
}

\author{
François Guillard', Pouya Golshan², Luming Shen, ${ }^{1}$ Julio R. Valdes ${ }^{2}$ and Itai Einav ${ }^{1,3 \star}$
}

\begin{abstract}
Brittle porous media exhibit a variety of irreversible patterns during densification, including stationary and moving compaction bands in rocks ${ }^{1-3}$, foams $^{4}$, cereal packs ${ }^{5}$ and snow ${ }^{6}$. We have recently found moving compaction bands in cereal packs ${ }^{5}$; similar bands have been detected in snow 6 . However, the question of generality remains: under what conditions can brittle porous media disclose other densification patterns? Here, using a new heuristic lattice spring model undergoing repeated crushing events, we first predict the possible emergence of new types of dynamic compaction; we then discover and confirm these new patterns experimentally in compressed cereal packs. In total, we distinguish three observed compaction patterns: short-lived erratic compaction bands, multiple oscillatory propagating compaction bands reminiscent of critical phenomena near phase transitions, and diffused irreversible densification. The manifestation of these three different patterns is mapped in a phase diagram using two dimensionless groups that represent fabric collapse and external dissipation.
\end{abstract}

Compaction of brittle porous media is of central importance in industry and science, and has many ramifications, from destruction waves during meteoritic impacts ${ }^{7}$ to the formation of density heterogeneities in pharmaceutical pills ${ }^{8}$ and permeability barriers in rocks ${ }^{9,10}$. Previous work on brittle porous media has generally revealed two forms of compaction patterns: stationary and oscillatory propagating compaction bands. Specifically, stationary compaction bands with localized intense volumetric strain rate have been frequently observed in a wide range of brittle porous materials, including rocks ${ }^{1-3}$, foams ${ }^{4}$ and honeycomb materials ${ }^{11}$. The formation of such bands has been rationalized mathematically using continuum models of viscoplasticity ${ }^{12,13}$ or breakage mechanics ${ }^{14,15}$, with the latter connecting the process directly to the physics of grain breakage and pore collapse.

The formation of oscillatory propagating compaction bands in porous media was discovered more recently by Valdes et al. ${ }^{5}$, via uniaxially confined compression experiments on puffed rice packs (Fig. 1a). Similar to stationary compaction bands, the material within these bands experienced high volumetric strain rates accommodated by severe grain breakage and pore collapse. By comparing experiments on material placed in containers with different boundary roughness, this work motivated a connection between compaction dynamics to how energy is dissipated outside.

More recently, similar experiments on dry foamy snow ${ }^{6}$ also revealed oscillatory propagating compaction bands, although with only one or two oscillations per test (this will be discussed later, in Fig. 2g-i). This form of compaction was captured using a


Figure 1 | Experimental and numerical configurations. a, Experimental configuration. $\mathbf{b}$, Lattice spring model. c, Force-displacement relation for the springs undergoing repetitive breakage events (at forces $F_{b r}^{i}$ ) during loading. Unloading follows the loading curve until the spring length equals the relaxed length $l_{i}$ that corresponds to the number of breakage events $i$; beyond that point the force is set to zero.

phenomenological continuum model for snow, with an assumed elastoplastic yield function, power-law density dependence, shearinduced bond failure, strength recovery due to sintering, and nonlocality of damage ${ }^{6}$. The need for these assumptions indicates that the underlying mechanisms that control the emergence of oscillatory compaction bands are not clear yet.

In this paper we present experiments that unfold novel compaction patterns in brittle porous media. We show that all the observed patterns can be explained using a simple lattice spring model. We then map the manifested patterns in terms of a phase diagram that covers system and material parameters, applicable for brittle porous media in general.

The experimental configuration is shown schematically in Fig. 1a. It consists of a transparent cylinder initially filled with puffed rice cereal to a height $H_{0}$. The material is then compressed from the top using a circular piston with a constant velocity $V$ up to approximately $50 \%$ strain $\epsilon=1-H / H_{0}$. During loading we record the side of the cylinder's shaft using a regular or high-speed video camera, depending on the piston velocity. Pictures are then processed using particle image velocimetry to analyse the material's velocity field. As the compression is purely axial, all velocities at a given height are averaged; thus, the axial velocity patterns are presented as a function of the axial depth and time.

Figure 2a,c,e shows the spatio-temporal plots of the local material velocity $v(z)$ normalized by the piston velocity $V$ for three different $V$. Clearly, the way the material densifies depends strongly on $V$. At intermediate $V$ (Fig. 2c), we recover the oscillatory propagating compaction bands found previously ${ }^{5}$. We notice that a single period of oscillation starts with a compaction band that

\footnotetext{
${ }^{1}$ Particles and Grains Laboratory, School of Civil Engineering, The University of Sydney, Sydney, New South Wales 2006, Australia. ${ }^{2}$ Geo-Innovations Research Lab, Department of Civil, Construction, and Environmental Engineering, San Diego State University, San Diego, California 92182, USA. ${ }^{3}$ Department of Civil, Environmental \& Geomatic Engineering, Faculty of Engineering Science, University College London, London WC1E 6BT, UK. *e-mail: itai.einav@sydney.edu.au
} 

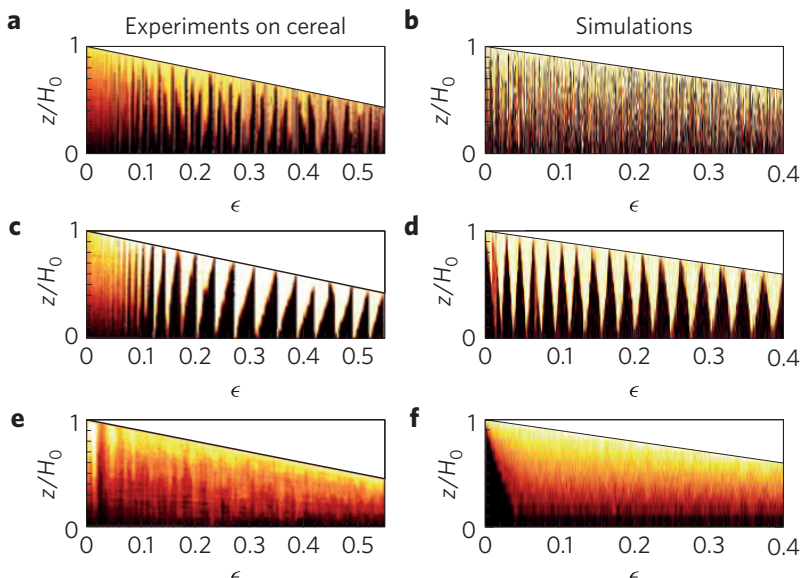
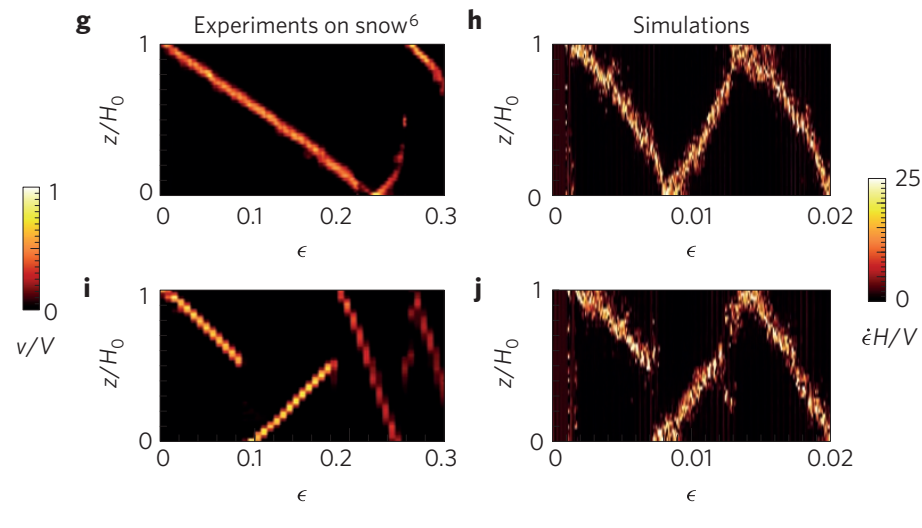

Figure 2 | Patterns of compaction from experiments and numerical model. a-f, Contours of local velocity $v$ normalized by piston velocity $V$. $\mathbf{a}, \mathbf{c}, \mathbf{e}$, Experiments with $V=0.013 \mathrm{~mm} \mathrm{~s}^{-1}, 1.3 \mathrm{~mm} \mathrm{~s}^{-1}$ and $300 \mathrm{~mm} \mathrm{~s}^{-1}$, respectively. b,d,f, Model simulations with $V=5 \times 10^{-6}, 2 \times 10^{-5}$ and $7 \times 10^{-5}$, respectively (with $k_{0}=2 \times 10^{-3}, F_{\text {br }}^{0}=2 \times 10^{-6}, \eta=1 \times 10^{-3}$ ). $\mathbf{g}$-j, Normalized local strain rate $\dot{\epsilon}$ in compaction experiments on snow for $V=1.125(\mathbf{g})$ and $5 \mathrm{~mm} \mathrm{~s}^{-1}$ (i) (adapted from ref. 6) and simulations with $V=2.45 \times 10^{-6}$ (h) and $2.5 \times 10^{-6}(\mathbf{j})\left(\right.$ for $\left.k_{0}=2.5 \times 10^{-4}, F_{b r}^{0}=2.5 \times 10^{-7}, \eta=2.5 \times 10^{-4}\right)$.

nucleates at the bottom; the band then propagates up until it reaches the top, and then re-emerges at the bottom, to start a new oscillation period. Such bands split the sample into an upper material block that moves down with a velocity close to the piston velocity $v(z) \sim V$ and a lower block with $v(z) \sim 0$; as shown by ref. 5, these two material blocks are separated by a narrow compaction band of intense velocity gradient. Under lower and higher piston velocities we unravel different forms of compaction. Under lower $V$ the material experiences short-lived and localized compaction appearing erratically in space (Fig. 2a). Under higher $V$ the material compacts almost homogeneously with $v(z) \simeq(V / H) z$.

In parallel to performing experiments, we developed a heuristic microstructural model to recover the oscillatory compaction patterns at the intermediate velocity. In carrying out computations, the model led us to detect the new patterns corresponding to the lower and higher velocities, which we then confirmed experimentally. Building on a spring lattice system, our model extends a previous quasi-static massless model that recovers stationary compaction bands ${ }^{16,17}$. Here, mass is included to tackle dynamic phenomena and the springs can take repetitive breakage events to model highly porous brittle material. The lattice is shown in Fig. 1b, and the response of the spring elements follow the forcelength diagram in Fig. 1c (see detailed information in Methods). The springs transmit elastic forces to the masses; these masses carry a further dissipative force given by global damping. Use of either local damping or friction is discussed in the Supplementary Information to further support the generality of our conclusions.

Figure $2 \mathrm{~b}, \mathrm{~d}, \mathrm{f}$ presents results from the lattice simulations with increasing $V$, which agree qualitatively with the experimental results of Fig. 2a,c,e. We note that, unlike the experimental oscillations in Fig. 2c, the theoretical oscillations in Fig. $2 \mathrm{~d}$ begin earlier and propagate symmetrically. Differences as such develop from unavoidable experimental heterogeneities, both through initial grain rearrangements and from statistical variation in grain strengths ${ }^{18}$. Indeed, although most experiments showed upward band propagation, a few others did exhibit either symmetrical or downward propagation.

We propose that such patterns are characteristics of general brittle porous media; the manifested compaction pattern depends on both material and system parameters. A first support for this generic claim is given in the Supplementary Information, which shows similar predictions for alternative force dissipation mechanisms (namely, similar patterns arise when one employs local damping or friction instead of global damping). A second support is given by comparing model simulations with experiments; this time on dry foamy snow, given by Barraclough et al. ${ }^{6}$ (see Fig. 2g,i), who adopted an experimental configuration similar to that of Valdes et al. ${ }^{5}$ (shown in Fig. 1a). Our simulations yield similar patterns already under much smaller strains (see Fig. 2h,j), including the featuring of incomplete upward and downward band propagations. The compliance and brittleness of puffed rice packs are much larger than those of snow, and, as such, puffed rice can accommodate many more oscillations; these properties allow us to identify the compaction patterns in Fig. 2a,e.

Next, we use the model to discover how breakage controls the various compaction patterns; an objective that is practically impossible to probe experimentally. For this purpose, we examine the breakage rate $\beta$ as a function of depth $z$ and strain $\epsilon$ by computing the average number of breakage events per spring between time steps. We normalize $\beta$ using a 'breakage time' $\tau_{\mathrm{bb}}^{0}$; that is, the typical time between two consecutive breakage events (see Methods).

Figure 3 shows the scaled breakage rate $\beta \tau_{\mathrm{br}}^{0}$ along $z$ and $\epsilon$ for five simulations with distinct identifiable patterns. Specifically, Fig. 3a,b,e correspond to Fig. 2a,c,e. It is clear by comparisons, especially between Figs $3 \mathrm{~b}$ and $2 \mathrm{c}$, that compaction band motions synchronize with breakage rate. There is almost no breakage outside the bands, whereas inside the breakage rate is the highest. Under the lowest imposed velocity (Fig. 3a), breakage develops irregularly in space and time, which explains why the corresponding velocity field reveals erratic and short-lived compaction bands. Under the highest velocity, the breakage develops almost continuously and uniformly (Fig. 3e), which explains why the corresponding velocity field is almost homogeneous. We detect further sub-patterns of oscillatory compaction bands-with bifurcation into more than a single oscillating band, as shown in Fig. $3 c$ (two fronts) and d (three fronts). These intricate velocity fields have not been observed experimentally yet; but this may be explained, as the range of parameters to observe such behaviours is small for experimental detection (as we show in Fig. 4).

Apart from the breakage time $\tau_{\mathrm{br}}$ mentioned above, two other timescales are identified from all the model parameters, including the elastic time $\tau_{\mathrm{el}}$ and the viscous time $\tau_{\eta}$. The elastic time defines the characteristic time required for an elastic wave to travel through the whole sample, and the viscous time denotes the typical time required for motion of mass $m$ to slow down as a result of the 
a



b


d
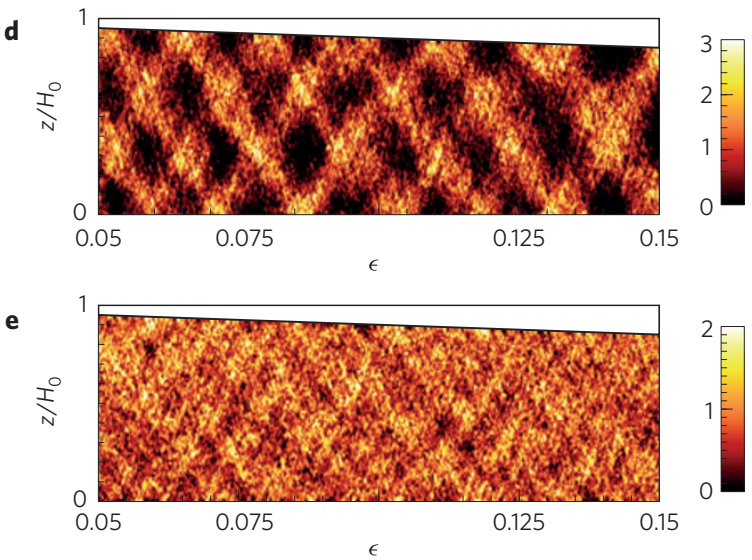

Figure $\mathbf{3}$ | Breakage patterns. a-e, Normalized breakage rate $\beta$ as a function of height $z$ and strain $\epsilon$ for various piston velocities: $V=5 \times 10^{-6}(\mathbf{a}), 2 \times 10^{-5}(\mathbf{b}), 4 \times 10^{-5}(\mathbf{c}), 5.2 \times 10^{-5}(\mathbf{d})$ and $7 \times 10^{-5}(\mathbf{e})$. All other parameters remain consistent with Fig. 2 .

global damping. In total, the system is defined in terms of the following times:

$$
\tau_{\mathrm{br}}=\frac{F_{\mathrm{br}}}{k l} \frac{H}{V} ; \quad \tau_{\mathrm{el}}=\frac{H}{l} \sqrt{\frac{m}{k}} ; \quad \tau_{\eta}=\frac{m}{\eta}
$$

where the index $i$ has been omitted to simplify the text. Given these three times we can define two non-dimensional groups via time ratios:

$$
B_{\mathrm{el}}=\frac{\tau_{\mathrm{br}}}{\tau_{\mathrm{el}}}=\frac{F_{\mathrm{br}}}{V \sqrt{k m}} ; \quad B_{\eta}=\frac{\tau_{\mathrm{br}}}{\tau_{\eta}}=\frac{F_{\mathrm{br}} H \eta}{k l V m}
$$

to be called the 'elasto-breakage number' and 'visco-breakage number', respectively.

Note that because the various parameters change during one simulation, $B_{\mathrm{el}}$ and $B_{\eta}$ change too. Specifically, over time the height $H$ decreases and the number of breakage events increases (i grows). Thus, $k_{i}$ and $F_{\mathrm{br}}^{i}$ increase as well. This may cause switches between the behavioural regimes, as observed in a few simulations. Therefore, the phase diagram in Fig. 4 collates only the first

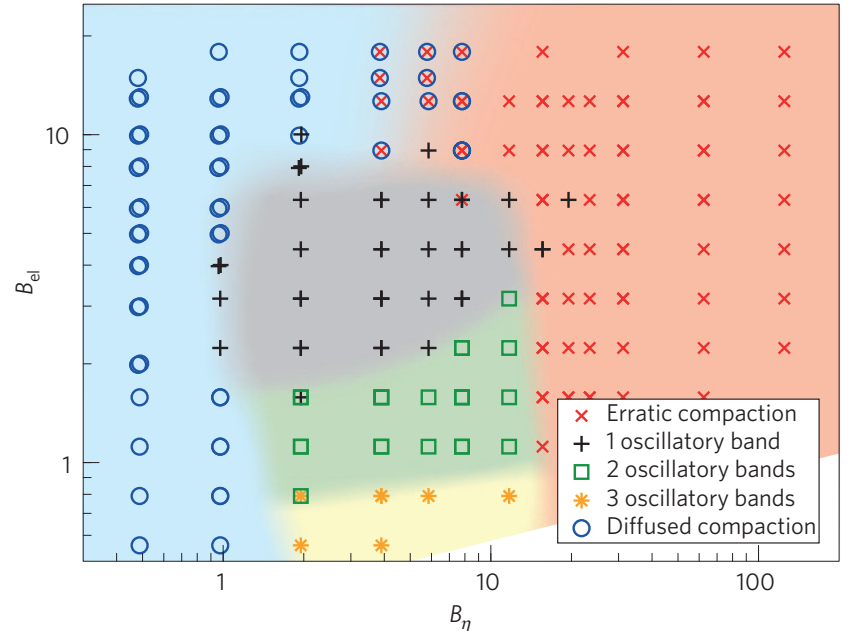

Figure 4 | Dimensionless phase diagram of compaction regimes. Shadings are added in the background to highlight the separation between the observed simulation patterns.

identifiable compaction regimes defined by the initial parameter values $\left(F_{\mathrm{br}}^{0}, k_{0}, l_{0}, H_{0}, \eta, m, V\right)$, in terms of $B_{\mathrm{el}}$ and $B_{\eta}$.

The compaction zones are short-lived and appear erratically when $B_{\eta}$ is high because the motion after a local breakage event has sufficient time to slow down by viscosity before further breakage, and because the steady state stress is essentially uniform throughout. Conversely, at very low $B_{\eta}$ there is not enough time for relaxation, and thus the whole sample undergoes crushing almost simultaneously. Between these two extreme regimes we find oscillatory propagating compaction bands with bifurcation dependent on the value of $B_{\mathrm{el}}$. As $B_{\mathrm{el}}$ gets smaller we move from a single oscillatory band to two or three bands, but the parametric resolution for capturing such bands narrows. This behaviour is reminiscent of critical phenomena near phase transitions.

In conclusion, this paper presents experiments that unfold compaction patterns inherent to brittle porous media, and a new heuristic spring lattice model that undertakes repetitive breakage events to explain those patterns. The model also captures other previously observed regimes of compaction in experiments on rocks, puffed rice packs and dry foamy snow. We note that during confined compaction, these materials undertake both internal fabric collapse (for example, breakage) and external dissipation (for example, viscous damping), as was underpinned by the model. We argue that although the physics that control these two processes may vary between materials, the mapping of the compaction patterns provided in this paper is generic for brittle porous media (see further support in the Supplementary Information).

Brittleness adds complexity to the dynamics of granular flows ${ }^{19}$ and can alter the propagation of density fronts often observed during the flow of loose, non-brittle granular media ${ }^{20-22}$. Finally, we recall another form of strain localization that occurs during the extension of metallic alloys-known as the Portevin-Le Châtelier (PLC) effect ${ }^{23-25}$. In this case, the motion of atomic impurities during the compression leads to a jerky flow of the material. The compaction band formed in the material (PLC type A) does not remain steady, but travels back and forth in the medium, leading to the observation of a stress drop when the band reaches one end. Therefore, as this is similar to the observations in Fig. $2 c, d$, our results in brittle porous media may provide a new perspective into the study of the PLC effect in alloys.

\section{Methods}

Methods and any associated references are available in the online version of the paper. 
Received 30 March 2015; accepted 1 July 2015; published online 10 August 2015

\section{References}

1. Olsson, W. A. Quasistatic propagation of compaction fronts in porous rock. Mech. Mater. 33, 659-668 (2001).

2. Vajdova, V. \& Wong, T. F. Incremental propagation of discrete compaction bands: Acoustic emission and microstructural observations on circumferentially notched samples of Bentheim. Geophys. Res. Lett. 30, 1775 (2003).

3. Baud, P., Klein, E. \& Wong, T. F. Compaction localization in porous sandstones: Spatial evolution of damage and acoustic emission activity. J. Struct. Geol. 26, 603-624 (2004)

4. Bastawros, A.-F., Bart-Smith, H. \& Evans, A. G. Experimental analysis of deformation mechanisms in a closed-cell aluminum alloy foam. J. Mech. Phys. Solids 48, 301-322 (1999).

5. Valdes, J. R., Fernandes, F. L. \& Einav, I. Periodic propagation of localized compaction in a brittle granular material. Granular Matter 14, 71-76 (2011).

6. Barraclough, T. W. et al. Propagating compaction bands in confined compression of snow: Experiment and Modelling. Preprint at http://arXiv.org/abs/1501.02184 (2015).

7. Love, S. G., Hörz, F. \& Brownlee, D. E. Target porosity effects in impact cratering and collisional disruption. Icarus 105, 216-224 (1993).

8. Wu, C. Y. et al. Modelling the mechanical behaviour of pharmaceutical powders during compaction. Powder Technol. 152, 107-117 (2005).

9. David, C., Wong, T. F., Zhu, W. \& Zhang, J. Laboratory measurement of compaction-induced permeability change in porous rocks: Implications for the generation and maintenance of pore pressure excess in the crust. Pure Appl. Geophys. 143, 425-456 (1994).

10. Olsson, W. A. Theoretical and experimental investigation of compaction bands in porous rock. J. Geophys. Res. 104, 7219-7228 (1999).

11. Papka, S. \& Kyriakides, S. Experiments and full-scale numerical simulations of in-plane crushing of a honeycomb. Acta Mater. 46, 2765-2776 (1998).

12. Rudnicki, J. \& Rice, J. Conditions for the localization of deformation in pressure-sensitive dilatant materials. J. Mech. Phys. Solids 23, 371-394 (1975).

13. Veveakis, E. \& Regenauer-Lieb, K. Cnoidal waves in solids. J. Mech. Phys. Solids 78, 231-248 (2015)

14. Das, A., Nguyen, G. D. \& Einav, I. The propagation of compaction bands in porous rocks based on breakage mechanics. J. Geophys. Res. 118 2049-2066 (2013).

15. Das, A. et al. A thermomechanical constitutive model for cemented granular materials with quantifiable internal variables. Part II-Validation and localization analysis. J. Mech. Phys. Solids 70, 382-405 (2014).
16. Katsman, R., Aharonov, E. \& Scher, H. Numerical simulation of compaction bands in high-porosity sedimentary rock. Mech. Mater. 37, 143-162 (2005)

17. Kale, S. \& Ostoja-Starzewski, M. Elastic-plastic-brittle transitions and avalanches in disordered media. Phys. Rev. Lett. 112, 045503 (2014).

18. McDowell, G. R. \& Humphreys, A. Yielding of granular materials. Granular Matter 4, 1-8 (2002).

19. Marks, B. \& Einav, I. A mixture of crushing and segregation: The complexity of grainsize in natural granular flows. Geophys. Res. Lett. 42, 274-281 (2015).

20. Wu, X. L., Måløy, K. J., Hansen, A., Ammi, M. \& Bideau, D. Why hour glasses tick. Phys. Rev. Lett. 71, 1363-1366 (1993).

21. Pennec, T. L., Ammi, M., Messager, J. C. \& Valance, A. Dynamics of density waves in a two dimensional funnel on an inclined plane. Eur. Phys. J. B 664, 657-664 (1999).

22. Mersch, E., Lumay, G., Boschini, F. \& Vandewalle, N. Effect of an electric field on an intermittent granular flow. Phys. Rev. E 81, 041309 (2010).

23. Penning, P. Mathematics of the Portevin-Le Chatelier effect. Acta Metall. 20, 1169-1175 (1972)

24. Kubin, L. P. \& Estrin, Y. The Portevin-Le Chatelier effect in deformation with constant stress rate. Acta Metall. 33, 397-407 (1985).

25. Lebedkina, T. A. \& Lebyodkin, M. A. Effect of deformation geometry on the intermittent plastic flow associated with the Portevin-Le Chatelier effect. Acta Mater. 56, 5567-5574 (2008).

\section{Acknowledgements}

I.E. and L.S. thank the Australian Research Council for support through project DP130101291. J.R.V. thanks the USA National Science Foundation for support through Grant CBET1336952. The authors also thank D. Griffani, M. Prokopenko and A. Sfriso for fruitful discussions.

\section{Author contributions}

F.G., I.E. and L.S. conceived the model. F.G. performed model simulations. J.R.V. and I.E. conceived the experiments. P.G., J.R.V. and F.G. performed the experiments. All the authors contributed to the writing of the manuscript.

\section{Additional information}

Supplementary information is available in the online version of the paper. Reprints and permissions information is available online at www.nature.com/reprints. Correspondence and requests for materials should be addressed to I.E.

\section{Competing financial interests}

The authors declare no competing financial interests. 


\section{Methods}

Numerical configuration. The lattice is a two-dimensional square lattice with initial cell lengths $l_{0}$ and masses $m$ at its vertices (see Fig. 1b). The springs transmit elastic forces to the masses; these masses carry a further dissipative force. We use a Verlet algorithm to integrate the mass motions at every time step $\Delta t$

The response of the spring elements follows the force-length diagram in Fig. 1c, which idealizes the compaction response of a single puffed rice grain sustaining multiple breakages. The growths in both the stiffness and breakage strength are linear and follow the rules $k_{i+1}=k_{i}+a k_{0}$ and $F_{\mathrm{br}}^{i+1}=F_{\mathrm{br}}^{i}+a F_{\mathrm{br}}^{0}$, with $a=0.01$ and $i$ being the number of breakage events. The dissipative force that acts on the masses is given either by global or local viscous damping, or by friction. Here we use global viscous damping to represent interactions with a background fluid at rest; at node $j$ this force is calculated by $\eta v_{j}$, where $\eta$ is the viscosity and $v_{j}$ is the nodal velocity. Use of either local damping or friction is discussed in the Supplementary Information to further support the generality of our conclusions.
The relaxed lengths of the springs are initially set to be the distance between nodes. Nodes at the bottom boundary are fixed, nodes along the sides can move only vertically, and nodes along the top move at constant velocity $V$. As the lattice compresses, at some stage the springs start to break. A coarse-graining process is carried out to extract the velocity field, following ref. 26. Results are presented non-dimensionally in terms of basic units of mass $[M]=m$, length $[L]=l_{0}$ and time $[T]=\Delta t$.

Definition of the breakage time. In the model, spring breakage occurs every local strain $F_{\mathrm{br}}^{i} / k_{i} l_{i}$ and, as the macroscopic strain rate $V / H$ in a non-breakable system is uniform with depth, we find $\tau_{\mathrm{br}}^{i}=\left(F_{\mathrm{br}}^{i} / k_{i} l_{i}\right)(H / V)$. Because the relaxed spring length, critical breakage force and spring stiffness vary in time and space, it is convenient to define the initial breakage time: $\tau_{\mathrm{br}}^{0}=\left(F_{\mathrm{br}}^{0} / k_{0} l_{0}\right)\left(H_{0} / V\right)$.

\section{References}

26. Glasser, B. J. \& Goldhirsch, I. Scale dependence, correlations, and fluctuations of stresses in rapid granular flows. Phys. Fluids 13, 407-420 (2001). 